\title{
Development of Ultradeformable Liposomes with Fatty Acids for Enhanced Dermal Rosmarinic Acid Delivery
}

\author{
Thirapit Subongkot ${ }^{1, *}$, Tanasait Ngawhirunpat ${ }^{2}$ and Praneet Opanasopit ${ }^{2}$ \\ 1 Pharmaceutical Innovations of Natural Products Unit (PhInNat), Department of Pharmaceutical Technology, \\ Faculty of Pharmaceutical Sciences, Burapha University, Chonburi 20131, Thailand \\ 2 Faculty of Pharmacy, Silpakorn University, Nakhon Pathom 73000, Thailand; \\ Ngawhirunpat_T@su.ac.th (T.N.); opanasopit_p@su.ac.th (P.O.) \\ * Correspondence: thirapi@go.buu.ac.th; Tel.: +66-38-102-610
}

Citation: Subongkot, T.;

Ngawhirunpat, T.; Opanasopit, P.

Development of Ultradeformable

Liposomes with Fatty Acids for

Enhanced Dermal Rosmarinic Acid

Delivery. Pharmaceutics 2021, 13, 404.

https://doi.org/10.3390/

pharmaceutics13030404

Academic Editor: Tom Anchordoquy

Received: 25 February 2021

Accepted: 13 March 2021

Published: 18 March 2021

Publisher's Note: MDPI stays neutral with regard to jurisdictional claims in published maps and institutional affiliations.

Copyright: (c) 2021 by the authors. Licensee MDPI, Basel, Switzerland. This article is an open access article distributed under the terms and conditions of the Creative Commons Attribution (CC BY) license (https:// creativecommons.org/licenses/by/ $4.0 /)$.

\begin{abstract}
This study aimed to develop ultradeformable liposomes (ULs) with fatty acids, namely, oleic, linoleic, and linolenic acid, to improve the skin penetration of rosmarinic acid. This study also investigated the vesicle-skin interaction and skin penetration pathway of ULs with fatty acids using the co-localization technique of multifluorescently labeled particles. The prepared ULs were characterized in terms of size, surface charge, size distribution, shape, $\%$ entrapment efficiency (\% EE), and \% loading efficiency (\% LE). The prepared ULs with fatty acids had an average particle size between $50.37 \pm 0.3$ and $59.82 \pm 17.3 \mathrm{~nm}$ with a size distribution within an acceptable range and exhibited a negative surface charge. The average \% EE and \% LE were 9 and 24.02, respectively. The in vitro skin penetration study found that ULs with oleic acid could significantly increase the skin penetration of rosmarinic acid compared to ULs. According to confocal laser scanning microscopy observations, this study suggested that UL vesicles attach to the skin before releasing the entrapped drug to penetrate the skin. These findings suggested that ULs with oleic acid penetrated the skin via the transfollicular pathway as a major penetration pathway.
\end{abstract}

Keywords: ultradeformable liposomes; fatty acids; rosmarinic acid; dermal drug delivery; skin penetration pathway

\section{Introduction}

Dermal drug delivery is the transportation of active compounds into the skin for various purposes, such as disease treatment as well as diagnosis and cosmetic application. The skin is the largest organ and consists of heterogeneous layers, including the epidermis, dermis, and subcutaneous tissue. However, the delivery of drugs into the skin is limited by the stratum corneum, which is the outermost layer of the skin. Rosmarinic acid is a polyphenolic compound that has various biological activities, such as anti-inflammatory [1], antioxidant, and antimelanogenic properties [2,3]. Due to its useful pharmacological properties, the delivery of rosmarinic acid into the skin is beneficial for skin health. Rosmarinic acid has a molecular weight of $360.3 \mathrm{~g} / \mathrm{mol}, \log$ partition coefficient $(\log \mathrm{P})$ of 1.82 , and pKa of 3.57 [4].

Theoretically, compounds that have $\log \mathrm{P}$ values between 1 and 3 with a molecular weight below 500 Daltons was considered to have good skin penetration properties [5]. However, at skin $\mathrm{pH}(\mathrm{pH} \sim 5)$ [6], rosmarinic acid exhibits low solubility due to very low ionization. According to the ex vivo penetration of rosmarinic acid using full thickness human skin, rosmarinic acid was detected only in epidermis whereas rosmarinic acid inside the dermis and receiver medium was not found [7]. Thus, rosmarinic acid has low skin penetration efficiency due to its physicochemical properties.

To increase the skin penetration of drugs, various strategies have been used, such as microemulsions [8], liposomes [9], ultrasound [10], nanofibers [11], niosomes [12], and magnetophoresis [13]. Liposomes are spherical phospholipid bilayer nanoparticles that 
can entrap both hydrophilic and lipophilic drugs. Ultradeformable liposomes (ULs) are a type of elastic liposome generated by the addition of edge activators into liposomes. This type of liposome has been shown to increase the skin penetration of various drugs [14-16]. According to the in vitro skin penetration study by Subongkot et al. [14], ULs with terpenes could increase the skin penetration of sodium fluorescein more than ULs. Thus, the addition of a skin penetration enhancer for ULs is necessary for better efficacy in enhancing the skin penetration of UL.

There have been many reports of various types of skin penetration enhancers, such as surfactants, urea, terpenes, alcohols, peptides, sulfoxides, glycols, and fatty acids [17]. Many fatty acids are generally recognized as safe (GRAS) and are approved by the Food and Drug Administration (FDA) as inactive ingredients in many products. Fatty acids can be classified into two groups: Saturated fatty acids and unsaturated fatty acids. There have been reports that unsaturated fatty acids show more efficacy than saturated fatty acids for skin penetration enhancement $[18,19]$. There was a report using fatty acids as an enhancer for pulmonary drug delivery [20]. However, there has been no report using unsaturated fatty acids as skin penetration enhancers for ULs. Therefore, this study aimed to improve the skin penetration of rosmarinic acid using ULs with unsaturated fatty acids and to investigate the skin penetration pathway of ultradeformable liposomes. Moreover, the physicochemical properties of various rosmarinic acid loaded liposomes such as particle size, size distribution, and droplets surface charge, were investigated.

\section{Materials and Methods}

\subsection{Materials}

Rosmarinic acid, rhodamine B base, and cholesterol were purchased from SigmaAldrich, St. Louis, MO, USA. Phospholipids (Phospholipon 90 G) were donated by Lipoid $\mathrm{GmbH}$, Ludwigshafen, Germany. Oleic acid and linoleic acid were purchased from Sigma-Aldrich, St. Louis, MO, USA. Alpha-linolenic acid (linolenic acid $>70 \%$ ) was purchased from Tokyo Chemical Industry Co., Ltd., Tokyo, Japan. Tween 20 was purchased from Ajax Finechem, Auckland, New Zealand. 1,2-Dihexadecanoyl-sn-glycero3-phosphoethanolamine triethylammonium salt (NBD-PE) was purchased from Thermo Fisher Scientific, Waltham, MA, USA. 4',6-Diamidino-2-phenylindole dihydrochloride (DAPI) was purchased from Invitrogen, Camarillo, CA, USA. All the other reagents were of analytical grade and commercially available.

\subsection{Preparation of Rosmarinic Acid Solution and Different Rosmarinic Acid-Loaded Liposomes}

The rosmarinic acid solution was prepared by weighing $6.39 \mathrm{mg}$ of rosmarinic acid accurately in a volumetric flask, adding $150 \mu \mathrm{L}$ of ethanol and stirring until dissolved. Then, water was added to adjust the volume to $5 \mathrm{~mL}$. Various formulations of rosmarinic acid-loaded liposomes are shown in Table 1. There have been several methods to prepare liposomes such as the thin film method and supercritical assisted technique [21]. In this study, liposomal formulations were prepared using the conventional thin film method. The rosmarinic acid stock solution was prepared by weighing $125 \mathrm{mg}$ of rosmarinic acid into a volumetric flask, and adjusting the volume to $5 \mathrm{~mL}$ using methanol. To prepare the phospholipid stock solution, $0.773 \mathrm{~g}$ of phospholipid was weighed into a glass vial, brought to $5 \mathrm{~mL}$ with 2:1 $\mathrm{v} / \mathrm{v}$ chloroform:methanol, and stirred until completely dissolved. The cholesterol stock solution was prepared by weighing $0.0618 \mathrm{~g}$ of cholesterol into a glass vial, and $8 \mathrm{~mL}$ of 2:1 $\mathrm{v} / \mathrm{v}$ chloroform:methanol was added and stirred until dissolved. 
Table 1. Composition of liposomal formulations.

\begin{tabular}{|c|c|c|c|c|c|c|}
\hline Formulations & $\begin{array}{c}\text { Rosmarinic } \\
\text { Acid } \\
(\% w / v)\end{array}$ & $\begin{array}{l}\text { Phospholipid } \\
\quad(\% \text { w/v) }\end{array}$ & $\begin{array}{l}\text { Cholesterol } \\
(\% \text { w/v) }\end{array}$ & $\begin{array}{l}\text { Tween } 20 \\
(\% w / v)\end{array}$ & $\begin{array}{l}\text { Fatty Acids } \\
(\% w / v)\end{array}$ & $\begin{array}{l}\text { Water } \\
(\mathrm{mL})\end{array}$ \\
\hline Conventional liposomes (CL) & 0.13 & 0.69 & 0.078 & - & - & qs 100 \\
\hline Ultradeformable liposomes (ULs) & 0.13 & 0.69 & 0.078 & 2 & - & qs 100 \\
\hline ULs with oleic acid & 0.13 & 0.69 & 0.078 & 2 & 0.5 & qs 100 \\
\hline ULs with linoleic acid & 0.13 & 0.69 & 0.078 & 2 & 0.5 & qs 100 \\
\hline ULs with linolenic acid & 0.13 & 0.69 & 0.078 & 2 & 0.5 & qs 100 \\
\hline
\end{tabular}

To prepare $5 \mathrm{~mL}$ of rosmarinic acid-loaded ULs with fatty acids, $256 \mu \mathrm{L}$ of rosmarinic acid stock solution, $250 \mu \mathrm{L}$ of phospholipid stock solution, and $500 \mu \mathrm{L}$ of cholesterol stock solution were pipetted into a test tube. The solvent was removed using a soft stream of nitrogen gas until the thin film occurred throughout the test tube. The obtained thin film was kept in the desiccator overnight. Then, the thin film was hydrated with water and vortexed by a vortex mixer until it was completely dispersed. Afterwards, the liposomal dispersion was filled into a glass vial containing a mixture of Tween 20 and each fatty acid and sonicated by placing the vial in an ice bath using a $3 \mathrm{~mm}$ diameter probe sonicator (Vibra-Cell Processors VCX 750, SONICS \& MATERIALS, INC, Newtown, CT, USA) for $30 \mathrm{~min}$. The energy and amplitude of the probe sonicator were set at 10,000 Joules and $20 \%$, respectively, throughout the sonication time.

Rosmarinic acid-loaded conventional liposomes and rosmarinic acid-loaded ULs were produced using the same method, as described above.

\subsection{Characterization of Liposomes}

\subsubsection{Particle Size, Polydispersity Index (PDI), and Surface Charge}

The average particle size, PDI, and zeta potential of various rosmarinic acid-loaded liposomes were determined using a dynamic light scattering (DLS) particle size analyzer (Zetasizer Nano-ZS, Malvern Instrument, Worcestershire, UK) equipped with a $4 \mathrm{~mW}$ $\mathrm{He}-\mathrm{Ne}$ laser at a scattering angle of $173^{\circ}$. The sample was diluted with an appropriate amount of water prior to each measurement. The measurement was performed under ambient conditions and in triplicate.

\subsubsection{Transmission Electron Microscopy (TEM)}

TEM was used to analyze the size and shape of liposomes. Each liposomal formulation was diluted with an appropriate amount of water and sonicated in a sonicator bath. A drop of diluted liposomes was pipetted onto the formvar-coated grid, stained with $2 \%$ uranyl acetate aqueous solution, and allowed to dry at room temperature. The samples were visualized by TEM (Philips Tecnai 20; FEI/Philips Electron Optics, Eindhoven, The Netherlands) at $80 \mathrm{kV}$.

\subsubsection{Entrapment Efficiency (\% EE) and Loading Efficiency (\% LE)}

The EE percentage was determined from the ratio between the amount of drug entrapped inside liposomes and total drug incorporated in liposomal formulation. Each liposomal formulation $(0.5 \mathrm{~mL})$ was pipetted into the sample reservoir of the centrifugal filter unit with a molecular weight cutoff of 3000 Daltons (Amicon Ultra-0.5, Merck KGaA, Darmstadt, Germany) and centrifuged at $4{ }^{\circ} \mathrm{C}$ at $10,000 \times \mathrm{g}$ for $1 \mathrm{~h}$. The filtrate in the retentate vial was removed, and $250 \mu \mathrm{L}$ of water was added to the sample reservoir followed by centrifugation at $4{ }^{\circ} \mathrm{C}$ at $10,000 \times g$ for $40 \mathrm{~min}$. Then, rosmarinic acid entrapped in liposomes was disrupted by the addition of $0.1 \% w / v$ Triton X-100 in the sample reservoir and centrifuged at $4{ }^{\circ} \mathrm{C}$ at $10,000 \times g$ for $10 \mathrm{~min}$. The obtained filtrate was analyzed by 
high-performance liquid chromatography (HPLC), and the \% EE was calculated from Equation (1):

$$
\% \mathrm{EE}=\left(R_{L} / R_{i}\right) \times 100
$$

$R_{L}$ is the amount of rosmarinic acid entrapped in liposomes and $R_{i}$ is the initial amount of rosmarinic acid added to the liposomes.

The \% LE was calculated using Equation (2):

$$
\% \operatorname{LE}=\left(R_{t} / L_{t}\right) \times 100
$$

$R_{t}$ is the amount of rosmarinic acid entrapped in liposomal formulation and $L_{t}$ is the amount of phospholipid and cholesterol added to the liposomal formulation.

\subsection{In Vitro Skin Penetration Study \\ 2.4.1. Skin Preparation}

The full-thickness skin used in this study was neonatal porcine skin from piglets that died of natural causes and was provided by a local pig farm in Nakhon Pathom Province (Nakhon Pathom, Thailand). The skin was obtained from approximately $1 \mathrm{~kg}$ of the abdominal part of a pig. The obtained skin was stored in a refrigerator at $-40^{\circ} \mathrm{C}$ until use. The muscle layer that might have adhered to the skin from the collection process was removed using a surgical blade. Prior to the experiment, the skin was thawed and washed with phosphate-buffered saline (PBS) to remove any contaminants before mounting in diffusion cells.

\subsubsection{Skin Penetration Study}

The skin penetration study of rosmarinic acid from various formulations was performed using a Franz diffusion cell apparatus. The skin was mounted between the donor and receiver compartments by turning the stratum corneum to the donor part. Each water jacketed diffusion cell was connected to a water circulating bath to maintain the temperature at $32{ }^{\circ} \mathrm{C}$. The donor part was filled with $2 \mathrm{~mL}$ of the tested formulations, while the receiver compartment was filled with PBS. The donor compartment and sampling port were covered with parafilm ${ }^{\circledR}$ to prevent evaporation. After the treatment for $6 \mathrm{~h}$, the tested formulation in the donor part was removed, and the skin was washed with PBS three times before removal from the diffusion cell. The treated skin was wiped with tissue paper, and stratum corneum layers were removed from the skin using the tape strip method according to the method, as described by Subongkot and Sirirak [22]. The skin was fixed on an aluminum tray containing hard paraffin wax, and stratum corneum was stripped with a $24 \mathrm{~mm}$ wide pressure-sensitive adhesive tape $\left(\mathrm{Scotch}^{\circledR}\right.$ Transparent Tape 500, 3M Co., Ltd., Bangkok, Thailand) 40 times. All the stripped tapes were kept in glass vials containing $10 \mathrm{~mL}$ of ethanol and placed in a sonicator bath for $15 \mathrm{~min}$. One milliter of the extracted ethanol was pipetted into the microcentrifuge tube and centrifuged at 11,180× $g$ at $25{ }^{\circ} \mathrm{C}$ for $15 \mathrm{~min}$ (Thermo Scientific ${ }^{\mathrm{TM}}$, model: Sorvall ${ }^{\mathrm{TM}}$ Legend ${ }^{\mathrm{TM}}$ XTR Centrifuge, Thermo Scientific ${ }^{\mathrm{TM}}$, Waltham, MA, USA). The obtained supernatant was analyzed for the rosmarinic acid content by HPLC.

The rosmarinic acid amount in the stratum corneum was calculated from Equation (3):

$$
\text { Drug amount in the stratum corneum }\left(\mu \mathrm{g} / \mathrm{cm}^{2}\right)=R s / S
$$

$R s$ is the amount of rosmarinic acid in the stratum corneum $(\mu \mathrm{g})$ and $S$ is the skin penetration area $\left(\mathrm{cm}^{2}\right)$.

The remaining skin from which the stratum corneum was stripped was cut into small fragments and placed into a screw cap glass vial containing $3 \mathrm{~mL}$ of ethanol. The vial was then placed in the sonicator bath for $15 \mathrm{~min}$. Then, $1 \mathrm{~mL}$ of the extracted ethanol was pipetted into a microcentrifuge tube and centrifuged at $11,180 \times g$ at $25^{\circ} \mathrm{C}$ for $15 \mathrm{~min}$. The obtained supernatant was analyzed for the rosmarinic acid content by HPLC. The 
rosmarinic acid amount in the viable epidermis and dermis was calculated from Equation (4):

$$
\text { Drug amount in the viable epidermis and dermis }\left(\mu \mathrm{g} / \mathrm{cm}^{2}\right)=R v / S
$$

$R v$ is the amount of rosmarinic acid in the viable epidermis and dermis $(\mu \mathrm{g})$ and $S$ is the skin penetration area $\left(\mathrm{cm}^{2}\right)$.

The enhancement ratio (ER) was calculated from Equation (5):

$$
\begin{gathered}
\mathrm{ER}=\text { Drug amount in the viable epidermis and dermis of } \\
\text { liposomal formulation/Drug amount in the viable } \\
\text { epidermis and dermis of rosmarinic acid solution }
\end{gathered}
$$

\subsection{Confocal Laser Scanning Microscopy (CLSM) Study}

To investigate the vesicle-skin interaction and skin penetration pathway of ultradeformable liposomes with fatty acids, the co-localization technique using multifluorescently labeled particles was utilized. Rhodamine B base $(\log P=1.95)$ which exhibits red fluorescent was chosen as the entrapped drug due to the similarity of $\log \mathrm{P}$ with rosmarinic acid. To identify the liposome particle, NBD-PE, a green fluorescent phospholipid surfactant was labelled. This study was performed using the formulation that provided the highest skin penetration enhancement of rosmarinic acid from Section 2.4.2.

\subsubsection{Preparation of Rhodamine B Base-Loaded NBD-PE-Labeled ULs}

NBD-PE was accurately weighed at $8.04 \mathrm{mg}$ and dissolved in $1 \mathrm{~mL}$ of 2:1 $\mathrm{v} / \mathrm{v}$ chloroform:methanol to prepare a stock solution. To prepare rhodamine B base-loaded NBD-PElabeled ULs, phospholipids and cholesterol were used at the same concentration as seen in Table 1 with the addition of $150 \mu \mathrm{L}$ of NBD-PE stock solution, and rhodamine B base was used at a concentration of $0.068 \% w / v$. Rhodamine B base-loaded NBD-PE-labeled ULs were prepared using the same process, as described in Section 2.2.

\subsubsection{In Vitro Skin Penetration Study}

Franz diffusion cell was used to evaluate the skin penetration of multifluorescently labeled ULs according to the method, as described in Section 2.4.2. One milliliter of rhodamine B base-entrapped NBD-PE-labeled ULs was filled in the donor part without the addition of the receiving medium. After $30 \mathrm{~min}$, the treated formulation in the donor part was removed, and the skin was washed with PBS to remove the excess dye prior to CLSM visualization.

\subsubsection{Skin Cross-Sectioning}

A small piece of treated skin from Section 2.5.2 was cross-sectioned by cryomicrotome (Leica 1850, Leica Instruments $\mathrm{GmbH}$, Nussloch, Germany). The frozen tissue was embedded in a tissue freezing medium and cross-sectioned into $5 \mu \mathrm{m}$ thick sections on a positively charged slide (Bio-Optica, Milan, Italy). The slide containing sectioned tissue was stained with $10 \mu \mathrm{g} / \mathrm{mL}$ DAPI solution for $5 \mathrm{~s}$ and immersed in water to remove the excess dye. The slide was allowed to dry at room temperature and mounted with a toluene-based synthetic resin mounting medium before being covered with a coverslip.

\subsubsection{CLSM Visualization}

An inverted confocal laser scanning microscope (Zeiss LSM 800-Airy scan, Carl Zeiss, Jena, Germany) was used to observe the treated skin from Section 2.5.2 by placing on a cover slip and turning the stratum corneum side to the $10 \times$ objective lens. In order to gain the sequential $x-z$ plane images, the skin was visualized using a $20 \times$ objective lens. The slides containing sectioned skin from Section 2.5 .3 were observed by a $10 \times$ objective lens. The microscope is equipped with four diode lasers with excitation wavelengths of 405, 488, 561 , and $640 \mathrm{~nm}$. The ZEN software (blue edition) was used to operate the microscope. 


\subsection{High-Performance Liquid Chromatography (HPLC) Analysis}

Rosmarinic acid was quantitatively analyzed by HPLC (Agilent 1260 infinity II LC system, Agilent Technology, Santa Clara, CA, USA) using a C18 reversed-phase column ( $4.6 \times 250 \mathrm{~mm}, 5 \mu \mathrm{m}$ particle size, VertiSep UPS C18, Vertical, Nonthaburi, Thailand). The mobile phase was acetonitrile: $0.5 \% v / v$ formic acid at a ratio of 30:70 v/v using a flow rate of $1 \mathrm{~mL} / \mathrm{min}$. The sample injection volume was $20 \mu \mathrm{L}$. The detection wavelength was $330 \mathrm{~nm}$. The standard curve of the rosmarinic acid solution was prepared in the concentration range of $1-1000 \mu \mathrm{g} / \mathrm{mL}$ with $\mathrm{R}^{2}=0.9998$. The limit of detection (LOD) and limit of quantification (LOQ) were 0.1 and $1 \mu \mathrm{g} / \mathrm{mL}$, respectively. The accuracy of this validated method was $99.12 \pm 0.27$. To determine the precision, the percent relative standard deviation was 1.52 .

\subsection{Statistical Analysis}

All the data were statistically analyzed using the one-way analysis of variance (ANOVA). Values of $p<0.05$ were considered statistically significant.

\section{Results and Discussion}

\subsection{Characterization of Liposomes}

The average particle size, PDI, and zeta potential of various rosmarinic acid-loaded liposomes are shown in Table 2. The prepared liposomes had particle sizes in the range of 50-130 nm. The average particle sizes of CL and ULs were 130 and $71 \mathrm{~nm}$, respectively. The average particle sizes of different ULs with fatty acids were in the range of 50-60 $\mathrm{nm}$. The average particle size of CL was significantly higher than that of the ULs, ULs with oleic acid, ULs with linoleic acid, and ULs with linolenic acid. Thus, the addition of Tween 20 could reduce the particle size of liposomes. The average particle size of ULs was significantly higher than that of ULs with linoleic acid and ULs with linolenic acid. The addition of linoleic acid and linolenic acid could reduce the particle size of ULs. There was no significant difference in particle size among ULs with oleic acid, ULs with linoleic acid, and ULs with linolenic acid.

Table 2. The average particle size, polydispersity index (PDI), and zeta potential of various rosmarinic acid-loaded liposomes.

\begin{tabular}{cccc}
\hline Formulations & Particle Size (nm) & PDI & Zeta Potential (mV) \\
\hline CL & $130 \pm 5.1$ & $0.40 \pm 0.07$ & $-2.89 \pm 0.37$ \\
\hline ULs & $71 \pm 7.5$ & $0.28 \pm 0.024$ & $-2.54 \pm 0.58$ \\
\hline ULs with oleic acid & $60 \pm 17.3$ & $0.32 \pm 0.1$ & $-18.03 \pm 0.35$ \\
\hline ULs with linoleic acid & $50 \pm 0.3$ & $0.24 \pm 0.008$ & $-14.87 \pm 0.86$ \\
\hline ULs with linolenic acid & $53 \pm 2.3$ & $0.27 \pm 0.014$ & $-13.20 \pm 0.70$ \\
\hline Each value represents the mean \pm standard deviation $(n=3)$ & &
\end{tabular}

Each value represents the mean \pm standard deviation $(n=3)$.

All liposomal formulations had PDIs lower than 0.4, indicating that the prepared liposomes had a size distribution within an acceptable range. The zeta potential of liposomal formulations ranged from -2.54 to $-18.03 \mathrm{mV}$. Phosphatidylcholine, a major constituent of phospholipids used in this study, is a zwitterionic molecule that has positively charged functional groups and negatively charged functional groups from choline and phosphate, respectively. Phospholipids have an isoelectric point between 6 and 6.7 [23]. The neutral $\mathrm{pH}$ of liposomes $(\mathrm{pH} \sim 7)$ is higher than the isoelectric point of phospholipids. Phosphatidyl choline showed a negative charge. Rosmarinic acid has a $\mathrm{pKa}=3.57$ and ionizes to negatively charged molecules at neutral $\mathrm{pH}$ in liposomes. The fatty acids incorporated in ULs also ionized to negatively charged molecules from their carboxylic group. Therefore, rosmarinic acid-loaded ULs with fatty acids exhibited a negative charge. The TEM images of each liposomal formulation are shown in Figure 1. The generated liposomes were round in shape and had nanometer-scale sizes. 

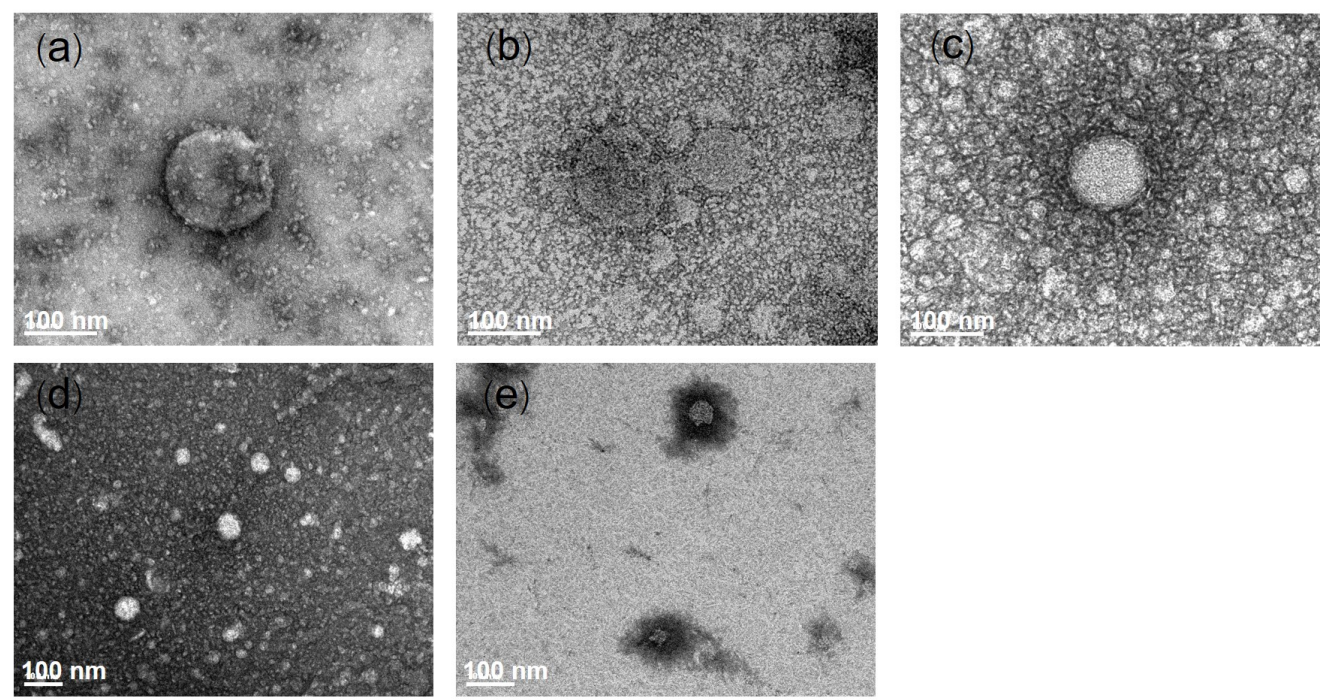

Figure 1. Transmission electron microscopy images of different rosmarinic acid-loaded liposomes: (a) Conventional liposomes (CL), (b) ultradeformable liposomes (ULs), (c) ULs with oleic acid, (d) ULs with linoleic acid, and (e) ULs with linolenic acid. The scale bar represents $100 \mathrm{~nm}$.

The effects of drug concentration added in the conventional liposome preparation on the EE and LE are shown in Figure 2. The increase in rosmarinic acid concentration from $5.55 \%$ to $16.64 \%$ of lipid weight led to a slight increase in EE. The EE reached the highest value $(9 \%)$ when the rosmarinic acid concentration was $16.64 \%$ of the lipid weight $(0.13 \%$ of rosmarinic acid in formulation). However, when the rosmarinic acid concentration rose above $16.64 \%$, the EE decreased. According to the study of entrapment efficiency of hydrophobic drugs, which were teicoplanin and rifampicin in liposomes prepared by the conventional thin film method. The maximum entrapment efficiency of teicoplanin and rifampicin were $23.4 \%$ and $15.5 \%$, respectively [24]. It is suggested that the low entrapment efficiency of the drug might result from the conventional method preparation. The \% LE slightly increased and reached the highest value at $8 \%$ when the rosmarinic acid concentration increased to $16.64 \%$ lipid weight. The \% LE decreased when the rosmarinic acid concentration rose above $16.64 \%$ of the lipid weight. Therefore, rosmarinic acid at a lipid weight of $16.64 \%$ was selected for the preparation of liposomes for further study.

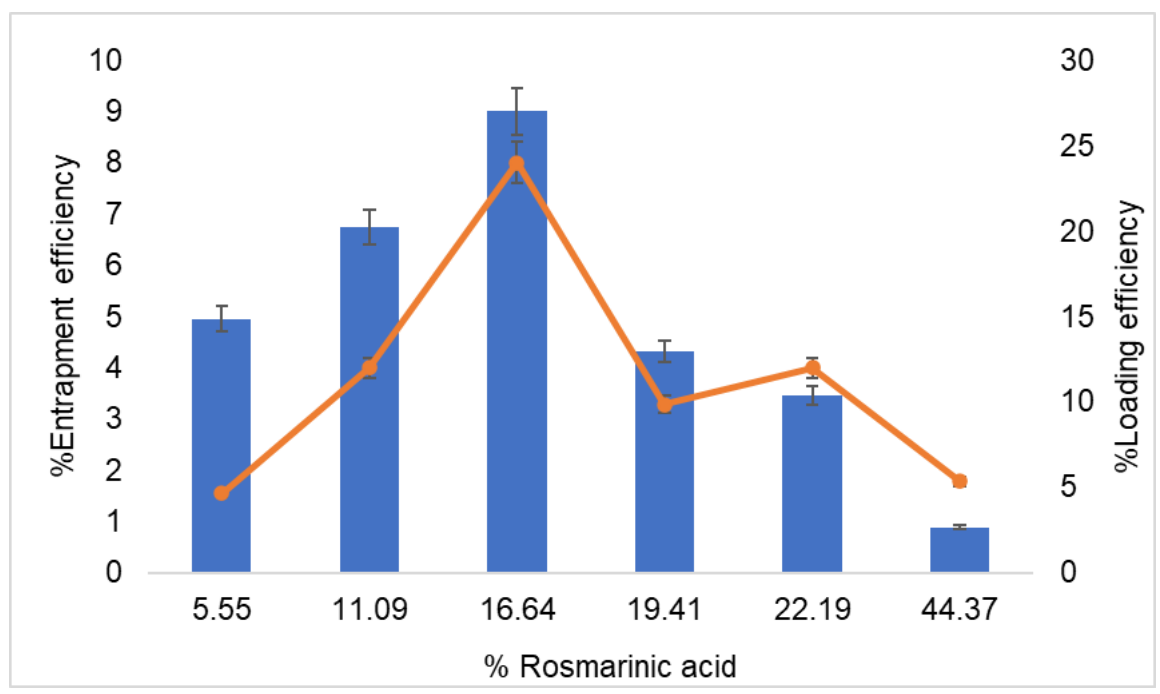

Figure 2. Effect of rosmarinic acid concentration (\% per lipid weight) on the \% entrapment efficiency $(\square)$ and loading efficiency (-). Each value represents the mean \pm standard deviation $(n=3)$. 


\subsection{In Vitro Skin Penetration Study}

The amounts of rosmarinic acid that penetrated into different layers of the skin are shown in Table 3. To compare the skin penetration enhancement efficacy of various formulations, only the amounts of rosmarinic acid in the viable epidermis and dermis were considered. The amounts of rosmarinic acid in ULs, ULs with $0.5 \%$ oleic acid, ULs with $0.5 \%$ linoleic acid, and ULs with $0.5 \%$ linolenic acid were significantly greater than those in the rosmarinic acid solution. However, only ULs with $0.5 \%$ oleic acid could improve the skin penetration of rosmarinic acid more than ULs. There was no significant difference in the amounts of rosmarinic acid among ULs with $0.5 \%$ oleic acid, ULs with $0.5 \%$ linoleic acid, and ULs with $0.5 \%$ linolenic acid.

Table 3. Skin penetration parameters of rosmarinic acid from each formulation.

\begin{tabular}{cccc}
\hline Formulations & $\begin{array}{c}\text { Stratum Corneum } \\
\left(\mathbf{m c g} / \mathbf{c m}^{2}\right)\end{array}$ & $\begin{array}{c}\text { Deeper Skin } \\
\left(\mathbf{m c g} / \mathbf{c m}^{2}\right)\end{array}$ & ER \\
\hline Solution & $2.70 \pm 0.53$ & $0.19 \pm 0.03$ & - \\
\hline ULs & $0.38 \pm 0.16$ & $0.43 \pm 0.22$ & 2.26 \\
\hline ULs with 0.5\% oleic acid & $1.13 \pm 0.10$ & $1.76 \pm 0.41^{*}$ & 9.26 \\
\hline ULs with 0.5\% linoleic acid & $0.46 \pm 0.16$ & $1.40 \pm 0.72$ & 7.37 \\
\hline ULs with 0.5\% linolenic acid & $1.91 \pm 0.64$ & $1.11 \pm 0.99$ & 5.84 \\
\hline
\end{tabular}

Each value represents the mean \pm standard deviation $(n=3)$. Deeper skin $=$ viable epidermis and dermis; ${ }^{*} p<$ 0.05 compared to the ULs.

Regarding the ER, ULs, ULs with $0.5 \%$ oleic acid, ULs with $0.5 \%$ linoleic acid, and ULs with $0.5 \%$ linolenic acid could increase the skin penetration of rosmarinic acid compared to the solution by $2.26-, 9.26-, 7.37-$, and 5.84 -fold, respectively. Thus, ULs with $0.5 \%$ oleic acid were chosen for further study.

The stratum corneum, the rate-limiting step in percutaneous absorption, is composed of corneocytes embedded in a lipid matrix, such as ceramide, triglycerides, cholesterol, and free fatty acids $[25,26]$. The barrier of the stratum corneum limits skin penetration resulting from intercellular lipid lamellae $[27,28]$ and keratin filaments inside corneocytes and corneodesmosomes that connect corneocytes between each layer of the stratum corneum [28]. According to the study by Subongkot et al. [29], ULs with d-limonene could increase the skin penetration of fluorescein sodium from degradation of corneodesmosomes, keratin filament denaturation, and intercellular lipid disruption. There is evidence showing that oleic acid could enhance the skin penetration by increasing the stratum corneum lipid fluidity and forming permeable defects inside intercellular lipids [30,31]. This study, therefore, suggested that the ULs with oleic acid could increase the skin penetration of rosmarinic acid through the mechanisms described above.

Among fatty acids, oleic acid is generally accepted as an effective skin penetration enhancer which can increase the skin penetration by penetrating to the stratum corneum and disturbing the lipid organization [31,32]. For linoleic acid, there was an evidence showing that linoleic acid had poor percutaneous absorption and must be improved using liposomes [33]. The skin penetration enhancement of rosmarinic acid from ULs with oleic acid might result from the skin penetration ability of oleic acid, in which oleic acid can penetrate the skin more than the other fatty acids.

\subsection{CLSM Study}

According to the in vitro skin penetration study, ULs with oleic acid could increase the highest amount of rosmarinic acid. ULs with oleic acid were, therefore, chosen to investigate the skin penetration pathway using the co-localization technique of multifluorescently labeled particles. Theoretically, there are three proposed skin penetration pathways, namely, the intercellular pathway, transcellular pathway, and transfollicular pathway [34-36]. 
The top view images of whole porcine skin treated with rhodamine B base-loaded NBD-PE-labeled ULs with oleic acid are shown in Figure 3. Rhodamine B base as an entrapped drug and NBD-PE, which represent UL particles, show red fluorescence and green fluorescence, as seen in Figure $3 a, b$, respectively. Red and green fluorescence was clearly seen at the hair follicles, indicating the localization of ULs via the transfollicular pathway. Surprisingly, Figure $3 a, b$ shows the same deposition pattern of red and green fluorescence. These data could be used to investigate the vesicle-skin interaction of ULs. This study hypothesized the release and attachment process of drug-loaded UL vesicles based on the possibility of fluorescence color deposition. If drug-loaded ULs attach to any part of the skin before releasing the entrapped drug, the red and green fluorescence will deposit within the same region. If the drug is released before the UL vesicles attach to the skin, the red and green fluorescence will not deposit in the same region. Regarding the same color deposition in Figure 3a,b, this study suggested that drug-loaded UL vesicles might attach to any part of the skin before releasing the entrapped drug into the skin.
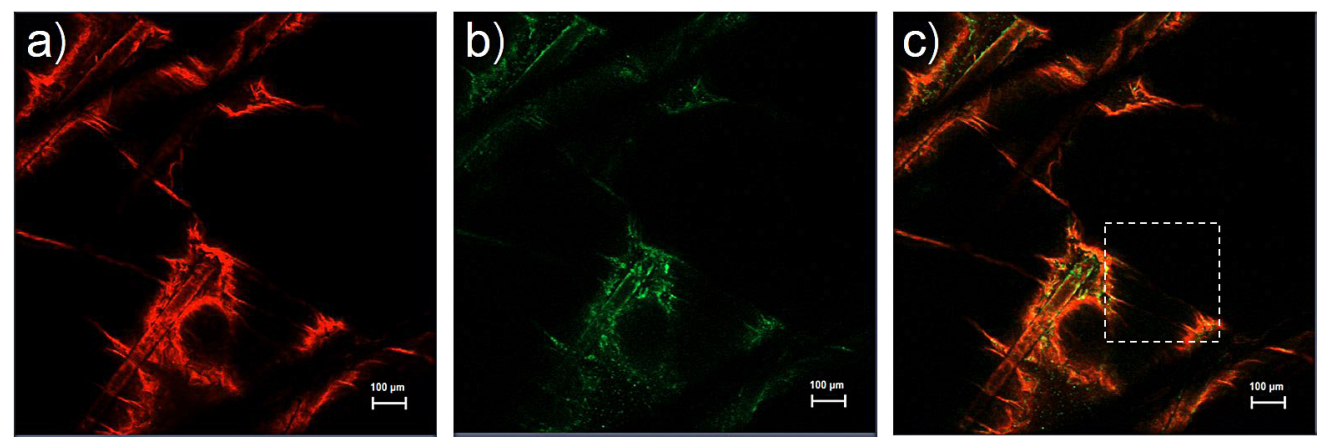

Figure 3. Top view images ( $x-y$ plane) of the skin treated with rhodamine B base-loaded NBD-PElabeled ultradeformable liposomes at $30 \mathrm{~min}$; (a) red fluorescence of rhodamine B base, (b) green fluorescence of NBD-PE, and (c) merged image of $(\mathbf{a}, \mathbf{b})$. The scale bar represents $100 \mu \mathrm{m}$.

The serial $x-z$ plane images of the marked area in Figure $3 c$ are shown in Figure $4 a$. Figure $4 \mathrm{~b}$ shows the intensity over the projection of the $z$-axis in Figure $4 \mathrm{a}$. Red fluorescence, green fluorescence, and merged images at the $55 \mu \mathrm{m}$ layer in Figure 4 are shown in Figure $5 \mathrm{a}-\mathrm{c}$, respectively. Figure $5 \mathrm{a}, \mathrm{b}$ shows different deposition patterns of red and green fluorescence. Many green fluorescence spots appeared in Figure $5 b$ but did not appear in Figure 5a. These green fluorescence spots indicated the existence of UL vesicles without the presence of entrapped drugs. This study suggested that UL vesicles attach to the skin surface before releasing the entrapped drugs for further penetration through the skin.

a)

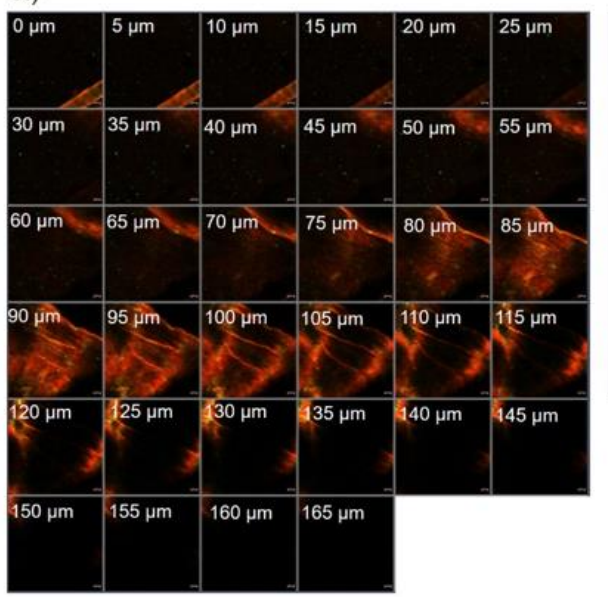

b)

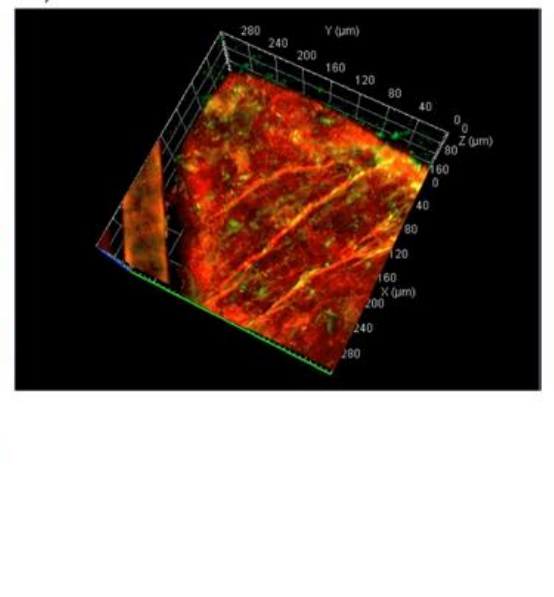

Figure 4. (a) The serial x-z plane images of the marked area in Figure 3c. The scale bar represents $20 \mu \mathrm{m},(\mathbf{b})$ intensity over the projection of $z$-axis in Figure $4 \mathrm{a}$. 

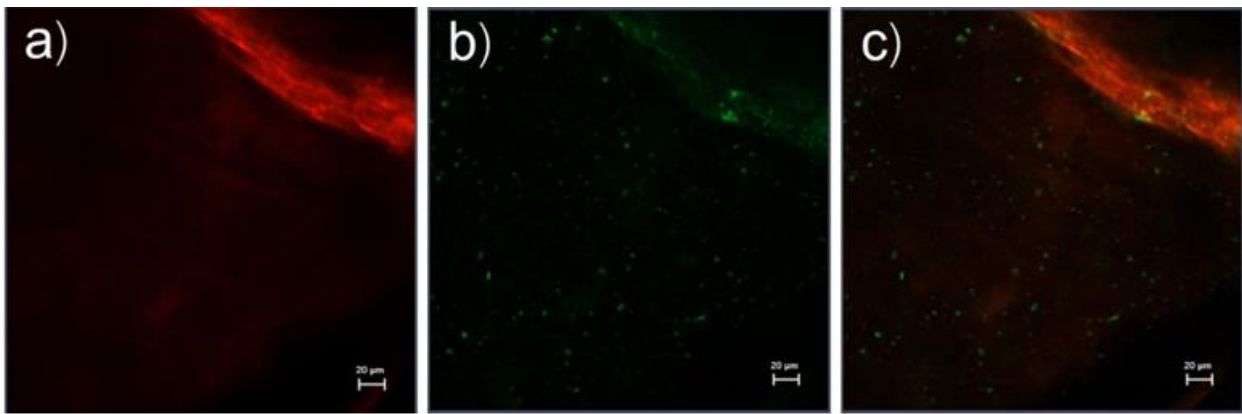

Figure 5. (a) Red fluorescence, (b) green fluorescence, and (c) merged image at the $55 \mu \mathrm{m}$ layer in Figure $4 \mathrm{a}$. The scale bar represents $20 \mu \mathrm{m}$.

Cross-sectional images of porcine skin treated with rhodamine B base-loaded NBDPE-labeled ULs are shown in Figure 6a-c. In Figure 6a, red fluorescence was clearly seen at the hair follicles more than the tissue region, indicating the penetration of UL vesicles via hair follicles. Figure $6 \mathrm{~b}, \mathrm{c}$ reveals green fluorescence, as seen in the white circles, indicating the penetration of UL vesicles into the hair follicles in which the entrapped drugs were already released to the surrounding tissues. Figure $6 b, c$ also illustrates the penetration of UL vesicles via hair follicles as a major skin penetration pathway. These findings agreed with a previous study in which ULs with d-limonene penetrated the skin through hair follicles as a major skin penetration pathway [34]. Although skin appendages occupy approximately $0.1 \%$ of the total skin surface area [37], this study revealed that the transfollicular pathway was the major pathway for the penetration of UL vesicles. The intercluster pathway was the other pathway of absorption into pig skin, as found by Carrer et al. [38]. The intercluster pathways were the canyons that surrounded the cluster of corneocytes. This canyon depth occurred from the stratum corneum surface to the dermis. As observed by two-photon microscopy, negatively charged Rh-PE-labeled liposomes were evidently found in the intercluster pathway. It is suggested that the intercluster pathway might also be responsible for the penetration of ULs with oleic acid.
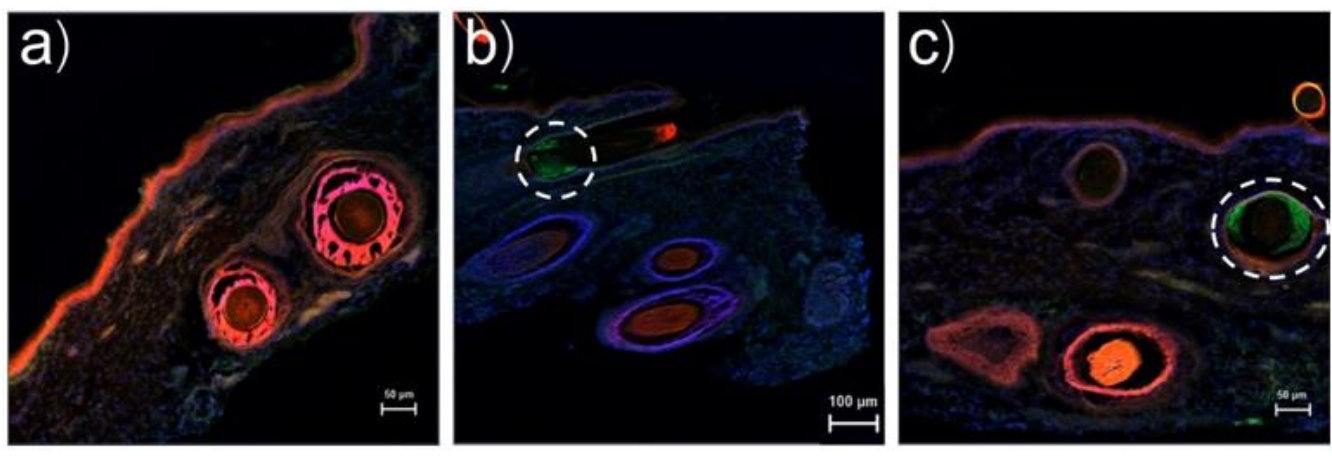

Figure 6. (a-c) Cross-sectional images of skin treated with rhodamine B base-loaded NBD-PE-labeled $\mathrm{UL}$ and stained with DAPI in different regions. The white circles indicate the presence of UL vesicles inside hair follicles in which the entrapped drugs were released.

\section{Conclusions}

ULs with fatty acids were successfully prepared using oleic acid, linoleic acid or linolenic acid at a concentration of $0.5 \% w / v$. The obtained ULs with fatty acids had a nanometer-scale size with size distribution within an acceptable range and negative surface charge. However, only ULs with oleic acid could significantly increase the skin penetration of rosmarinic acid compared to the control. The CLSM study using the co-localization technique suggested that ULs with oleic acid might attach to any part of the skin before releasing the entrapped drug to further penetrate the skin. ULs with oleic acid might penetrate the skin via the transfollicular pathway as a major pathway, while intercellular 
and transcellular pathways are minor penetration pathways. Penetration through the skin via hair follicles by passing the stratum corneum might also be the main mechanism for skin penetration enhancement of ULs with oleic acid.

Author Contributions: Conceptualization, investigation, writing—review and editing, T.S.; manuscript revision, T.N.; Funding acquisition, manuscript editing, P.O. All authors have read and agreed to the published version of the manuscript.

Funding: This research was supported by the Thailand Science Research and Innovation (TSRI) through the Research Team Promotion Grant (RTA6180003) and the Faculty of Pharmaceutical Sciences, Burapha University (Grant no. Rx11/2564).

Institutional Review Board Statement: Not applicable.

Informed Consent Statement: Not applicable.

Data Availability Statement: The data presented in this study is contained within this article.

Acknowledgments: The authors would like to thank the Pharmaceutical Innovations of Natural Products Unit, Faculty of Pharmaceutical Sciences, Burapha University, for providing support. The authors also would like to thank Lipoid GmbH for donating the Phospholipon $90 \mathrm{G}$ sample.

Conflicts of Interest: The authors declare no conflict of interest. The funders had no role in the design of the study; in the collection, analyses, or interpretation of data; in the writing of the manuscript, or in the decision to publish the results.

$\begin{array}{ll}\text { Abbreviations } \\ \text { CL } & \text { Conventional liposomes } \\ \text { ULs } & \text { Ultradeformable liposomes } \\ \text { EE } & \text { Entrapment efficiency } \\ \text { LE } & \text { Loading efficiency } \\ \text { ER } & \text { Enhancement ratio }\end{array}$

\section{References}

1. Rocha, J.; Eduardo-Figueira, M.; Barateiro, A.; Fernandes, A.; Brites, D.; Bronze, R.; Duarte, C.M.; Serra, A.T.; Pinto, R.; Freitas, M.; et al. Anti-inflammatory effect of rosmarinic acid and an extract of Rosmarinus officinalis in rat models of local and systemic inflammation. Basic Clin. Pharmacol. Toxicol. 2015, 116, 398-413. [CrossRef]

2. Ding, H.-Y.; Chou, T.-H.; Liang, C.-H. Antioxidant and antimelanogenic properties of rosmarinic acid methyl ester from Origanum vulgare. Food Chem. 2010, 123, 254-262. [CrossRef]

3. Fujimoto, A.; Shingai, Y.; Nakamura, M.; Maekawa, T.; Sone, Y.; Masuda, T. ChemInform Abstract: A Novel Ring-Expanded Product with Enhanced Tyrosinase Inhibitory Activity from Classical Fe-Catalyzed Oxidation of Rosmarinic Acid, a Potent Antioxidative Lamiaceae Polyphenol. Bioorg. Med. Chem. Lett. 2011, 42, 7393-7396. [CrossRef]

4. Available online: https:// pubchem.ncbi.nlm.nih.gov/compound/Rosmarinic-acid (accessed on 1 December 2020).

5. Naik, A.; Kalia, Y.N.; Guy, R.H. Transdermal drug delivery: Overcoming the skin's barrier function. Pharm. Sci. Technol. Today 2000, 3, 318-326. [CrossRef]

6. Lambers, H.; Piessens, S.; Bloem, A.; Pronk, H.; Finkel, P. Natural skin surface pH is on average below 5, which is beneficial for its resident flora. Int. J. Cosmet. Sci. 2006, 28, 359-370. [CrossRef] [PubMed]

7. Stelmakiene, A.; Ramanauskienè, K.; Briedis, V. Release of rosmarinic acid from semisolid formulations and its penetration through human skin ex vivo. Acta Pharm. 2015, 65, 199-205. [CrossRef]

8. Hathout, R.M.; Nasr, M. Transdermal delivery of betahistine hydrochloride using microemulsions: Physical characterization, biophysical assessment, confocal imaging and permeation studies. Colloids Surfaces B Biointerfaces 2013, 110, 254-260. [CrossRef] [PubMed]

9. Lee, M.-H.; Lee, K.-K.; Park, M.-H.; Hyun, S.-S.; Kahn, S.-Y.; Joo, K.-S.; Kang, H.-C.; Kwon, W.-T. In vivo anti-melanogenesis activity and in vitro skin permeability of niacinamide-loaded flexible liposomes (Bounsphere ${ }^{\mathrm{TM}}$ ). J. Drug Deliv. Sci. Technol. 2016, 31, 147-152. [CrossRef]

10. Liao, A.-H.; Lu, Y.-J.; Hung, C.-R.; Yang, M.-Y. Efficacy of transdermal magnesium ascorbyl phosphate delivery after ultrasound treatment with microbubbles in gel-type surrounding medium in mice. Mater. Sci. Eng. C 2016, 61, 591-598. [CrossRef]

11. Song, J.; Fan, X.; Shen, Q. Daidzein-loaded nanostructured lipid carriers-PLGA nanofibers for transdermal delivery. Int. J. Pharm. 2016, 501, 245-252. [CrossRef] 
12. Pando, D.; Matos, M.; Gutiérrez, G.; Pazos, C. Formulation of resveratrol entrapped niosomes for topical use. Colloids Surfaces B Biointerfaces 2015, 128, 398-404. [CrossRef]

13. Sammeta, S.M.; Repka, M.A.; Murthy, S.N. Magnetophoresis in combination with chemical enhancers for transdermal drug delivery. Drug Dev. Ind. Pharm. 2011, 37, 1076-1082. [CrossRef] [PubMed]

14. Subongkot, T.; Duangit, S.; Rojanarata, T.; Opanasopit, P.; Ngawhirunpat, T. Ultradeformable liposomes with terpenes for delivery of hydrophilic compound. J. Liposome Res. 2012, 22, 254-262. [CrossRef] [PubMed]

15. Cristiano, M.C.; Froiio, F.; Spaccapelo, R.; Mancuso, A.; Nisticò, S.P.; Udongo, B.P.; Fresta, M.; Paolino, D. Sulforaphane-Loaded Ul-tradeformable Vesicles as A Potential Natural Nanomedicine for the Treatment of Skin Cancer Diseases. Pharmaceutics 2019, 12, 6. [CrossRef]

16. Lin, H.; Xie, Q.; Huang, X.; Ban, J.; Wang, B.; Wei, X.; Chen, Y.; Lu, Z. Increased skin permeation efficiency of imperatorin via charged ultradeformable lipid vesicles for transdermal delivery. Int. J. Nanomed. 2018, 831-842. [CrossRef]

17. Williams, A.C.; Barry, B.W. Penetration enhancers. Adv. Drug Deliv. Rev. 2012, 64, 603-618. [CrossRef]

18. Aungst, B.J.; Rogers, N.J.; Shefter, E. Enhancement of naloxone penetration through human skin in vitro using fatty acids, fatty alcohols, surfactants, sulfoxides and amides. Int. J. Pharm. 1986, 33, 225-234. [CrossRef]

19. Aungst, B.J. Structure/Effect Studies of Fatty Acid Isomers as Skin Penetration Enhancers and Skin Irritants. Pharm. Res. 1989, 6, 244-247. [CrossRef] [PubMed]

20. Ghadiri, M.; Canney, F.; Pacciana, C.; Colombo, G.; Young, P.M.; Traini, D. The use of fatty acids as absorption enhancer for pul-monary drug delivery. Int. J. Pharm. 2018, 541, 93-100. [CrossRef]

21. Trucillo, P.; Campardelli, R.; Reverchon, E. Antioxidant loaded emulsions entrapped in liposomes produced using a supercritical assisted technique. J. Supercrit. Fluids 2019, 154, 104626. [CrossRef]

22. Subongkot, T.; Sirirak, T. Development and skin penetration pathway evaluation of microemulsions for enhancing the dermal delivery of celecoxib. Colloids Surfaces B Biointerfaces 2020, 193, 111103. [CrossRef]

23. Chain, E.; Kemp, I. The isoelectric points of lecithin and sphingomyelin. Biochem. J. 1934, 28, 2052-2055. [CrossRef]

24. Gomez, A.G.; Syed, S.; Marshall, K.; Hosseinidoust, Z. Liposomal Nanovesicles for Efficient Encapsulation of Staphylococcal Anti-biotics. ACS Omega 2019, 4, 10866-10876. [CrossRef] [PubMed]

25. Lampe, M.A.; Burlingame, A.L.; Whitney, J.; Williams, M.L.; Brown, B.E.; Roitman, E.; Elias, P.M. Human stratum corneum lipids: Char-acterization and regional variations. J. Lipid. Res. 1983, 24, 120-130. [CrossRef]

26. van Smeden, J.; Bouwstra, J.A. Stratum Corneum Lipids: Their Role for the Skin Barrier Function in Healthy Subjects and Atopic Dermatitis Patients. Curr. Probl. Dermatol. 2016, 49, 8-26. [PubMed]

27. Harada, K.; Murakami, T.; Yata, N.; Yamamoto, S. Role of Intercellular Lipids in Stratum Corneum in the Percutaneous Permeation of Drugs. J. Investig. Dermatol. 1992, 99, 278-282. [CrossRef]

28. Sheu, H.M.; Lee, J.Y.; Chai, C.Y.; Kuo, K.W. Depletion of stratum corneum intercellular lipid lamellae and barrier function abnor-malities after long-term topical corticosteroids. Br. J. Dermatol. 1997, 136, 884-890. [CrossRef]

29. Ngawhirunpat, T.; Subongkot, T.; Rojanarata, T.; Opanasopit, P.; Pamornpathomkul, B. Investigation of the mechanism of enhanced skin penetration by ultradeformable liposomes. Int. J. Nanomed. 2014, 9, 3539-3550. [CrossRef]

30. Golden, G.M.; McKie, J.E.; Potts, R.O. Role of Stratum Corneum Lipid Fluidity in Transdermal Drug Flux. J. Pharm. Sci. 1987, 76, 25-28. [CrossRef]

31. Ongpipattanakul, B.; Burnette, R.R.; Potts, R.O.; Francoeur, M.L. Evidence that Oleic Acid Exists in a Separate Phase Within Stratum Corneum Lipids. Pharm. Res. 1991, 8, 350-354. [CrossRef]

32. Lane, M.E. Skin penetration enhancers. Int. J. Pharm. 2013, 447, 12-21. [CrossRef]

33. Shigeta, Y.; Imanaka, H.; Ando, H.; Ryu, A.; Oku, N.; Baba, N.; Makino, T. Skin Whitening Effect of Linoleic Acid Is Enhanced by Liposomal Formulations. Biol. Pharm. Bull. 2004, 27, 591-594. [CrossRef] [PubMed]

34. Subongkot, T.; Wonglertnirant, N.; Songprakhon, P.; Rojanarata, T.; Opanasopit, P.; Ngawhirunpat, T. Visualization of ultradeformable liposomes penetration pathways and their skin interaction by confocal laser scanning microscopy. Int. J. Pharm. 2013, 441, 151-161. [CrossRef] [PubMed]

35. Sala, M.; Diab, R.; Elaissari, A.; Fessi, H. Lipid nanocarriers as skin drug delivery systems: Properties, mechanisms of skin interactions and medical applications. Int. J. Pharm. 2018, 535, 1-17. [CrossRef] [PubMed]

36. Marwah, H.; Garg, T.; Goyal, A.K.; Rath, G. Permeation enhancer strategies in transdermal drug delivery. Drug Deliv. 2016, 23, 564-578. [CrossRef] [PubMed]

37. Moser, K.; Kriwet, K.; Naik, A.; Kalia, Y.N.; Guy, R.H. Passive skin penetration enhancement and its quantification in vitro. Eur. J. Pharm. Biopharm. 2001, 52, 103-112. [CrossRef]

38. Carrer, D.C.; Vermehren, C.; Bagatolli, L.A. Pig skin structure and transdermal delivery of liposomes: A two photon microscopy study. J. Control. Release 2008, 132, 12-20. [CrossRef] [PubMed] 